\title{
Multiwavelength Observations of NGC7027
}

\author{
I. Bains ${ }^{1}$, M. Bryce ${ }^{1}$, A. Pedlar ${ }^{2}$, T. W. B. Muxlow ${ }^{2}$, P. Thomasson ${ }^{2}$ and \\ G. Mellema ${ }^{3}$ \\ ${ }^{1}$ Astronomy Group, University of Manchester; ${ }^{2}$ Jodrell Bank, University of Manchester; \\ ${ }^{3}$ Stockholm Observatory
}

We have made multi-wavelength observations of the young planetary nebula NGC 7027. The C-band continuum radio map shown in Figure 1 was obtained from the combination of MERLIN and VLA ${ }^{1}$ observations (M. Bryce et al., 1996). This map has the highest spatial resolution of any radio images of this object to date and has been compared with the HST archive picture of NGC7027, taken using WFPC2 with a $400 \AA$ wide F547N filter, to reveal the differences in the emission at the disparate wavelengths. The two maps, while being superficially different, show emission regions that are similar in size. The contours of radio brightness often coincide with prominent optical features.

The L-band continuum radio map also shown in Figure 1 was obtained from MERLIN data alone (I. Bains et al., paper in preparation). It is unfinished and therefore contains noise artefacts and sidelobe structure, but the emission region can be seen to be of a similar size to that of the older C-band map and the bright knot in the NW sector of the shell is again prominent.

To complement the radio data, spatially resolved long slit spectra of the nebula were obtained, with a resolution of $6 \mathrm{~km} / \mathrm{s}$, using the Utrecht echelle spectrograph on the William Herschel Telescope (I. Bains et al., paper in preparation). The optical emission lines of [NII] $6548 \AA$ and [OIII] $5007 \AA$ trace velocity ellipses characteristic of an expanding shell of emission, the [OIII] emission lying within the [NII] shell. The ellipse seen when the long slit was placed along the major axis of the nebula clearly shows the SW of the shell as being redshifted and the NE blueshifted, indicative of a bipolar morphology. The placement of the slit in an E-W orientation over the bright knot seen in the NW of the shell on the radio maps, reveals a broken ellipse that appears bipolar in [NII] but not in [OIII].

\section{REFERENCES}

Bryce, M., Pedlar, A., Muxlow, T. W. B., Thomasson, P. \& Mellema, G., 1996, MNRAS, in press Bains, I., Bryce, M., Pedlar, A., Muxlow, T. W. B., Thomasson, P. \& Mellema, G., in preparation

${ }^{1}$ Combined A- and B-array data were used for better uv-coverage; the B-array data were supplied by Terzian. 


\section{Envelopes}
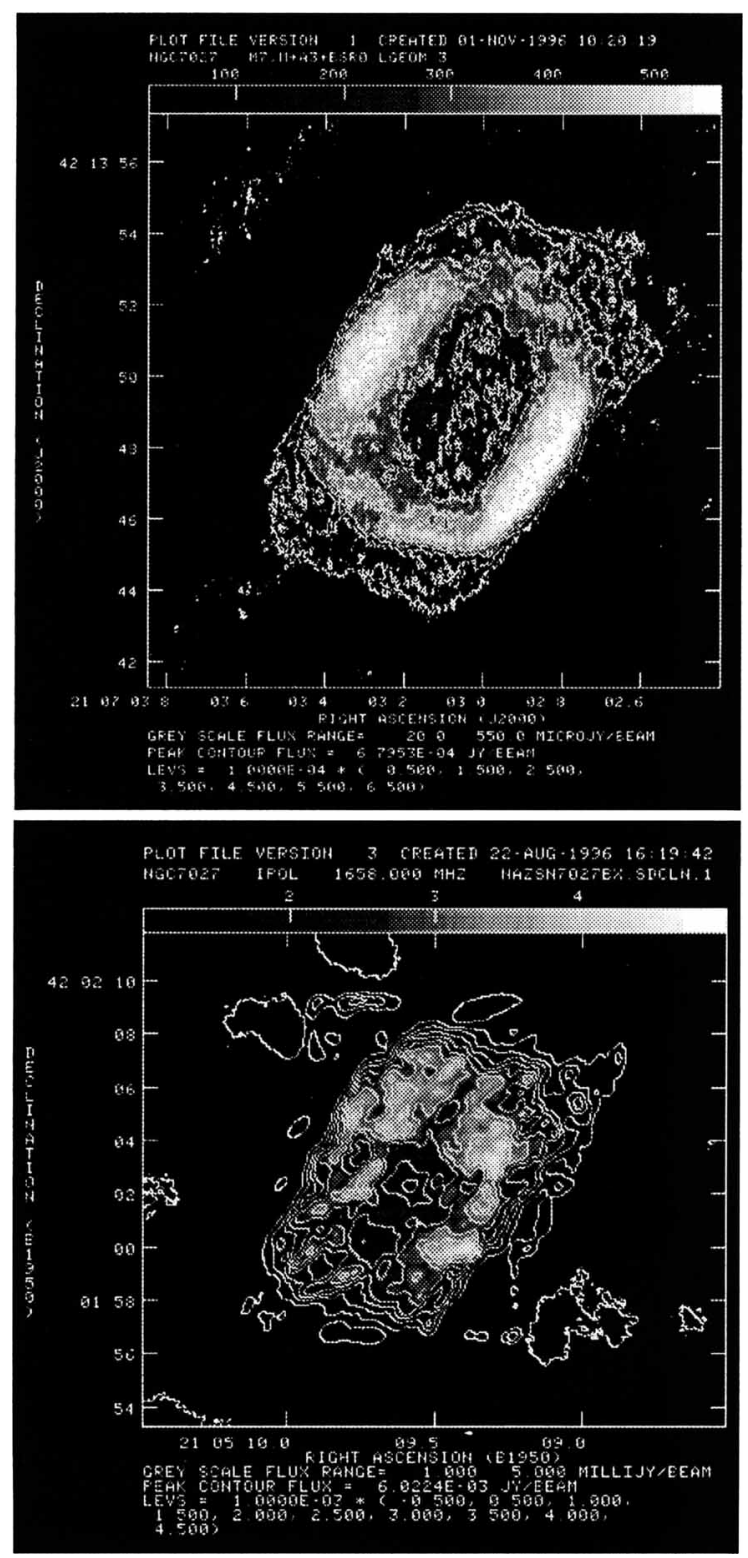

Figure 1: (top) The C-band continuum radio map derived from MERLIN and VLA data, with a restoring beamsize of $62 \times 48$ mas; (bottom) The L-band continuum MERLIN radio map made with a restoring beamsize of $390 \times 310$ mas. 